\title{
Intralobar Pulmonary Sequestration with Rete Mirabile as Part of Its Systemic Artery
}

\author{
Patrick Mailleux ${ }^{1}$, Marylène Clausse ${ }^{2}$ \\ ${ }^{1}$ Department of Imaging, Clinique St Luc, Bouge, Belgium \\ ${ }^{2}$ Department of Oncology, Internal Medicine, Clinique St Luc, Bouge, Belgium \\ Email: p.mailleux@gmail.com
}

How to cite this paper: Mailleux, P. and Clausse, M. (2020) Intralobar Pulmonary Sequestration with Rete Mirabile as Part of Its Systemic Artery. Open Journal of Medical Imaging, 10, 89-95.

https://doi.org/10.4236/ojmi.2020.102008

Received: April 29, 2020

Accepted: May 16, 2020

Published: May 19, 2020

Copyright () 2020 by author(s) and Scientific Research Publishing Inc. This work is licensed under the Creative Commons Attribution International License (CC BY 4.0).

http://creativecommons.org/licenses/by/4.0/ (c) (i) Open Access

\begin{abstract}
Intralobar sequestration (ILS) is a complex lung lesion where part of a lower lobe lacks communication with the tracheobronchial tree and receives an aberrant arterial blood supply from the systemic circulation. That systemic artery is usually large, originating from the thoracic aorta or the abdominal aorta and its upper branches. We describe a case of ILS where a large systemic artery coming up from below the diaphragm is formed by the convergence of many very small serpiginous arteries coming up from the area of the celiac trunk and common hepatic artery: a kind of "rete mirabile". This peculiar morphology was not described before.
\end{abstract}

\section{Keywords}

CT, Aberrant Systemic Artery, Congenital Anomalous Artery, Pulmonary Sequestration, Rete Mirabile

\section{Introduction}

Pulmonary sequestration is a congenital pathology where part of the lung, usually in the lower lobes, has no connection with the central airways, and an arterial supply coming from a systemic origin. It is called an intra-lobar sequestration (ILS) when the abnormal lung segment lies in the same pleural sac, while it is an extra-lobar sequestration (ELS) when exists a separate pleural cavity. The large feeding artery having a systemic origin is often the clue to the diagnosis. In the patient, a very peculiar finding is the appearance of the abnormal systemic artery, which is formed by the confluence of a meshwork of tiny arteries coming up from the area of the celiac trunk. 


\section{Case Report}

A 62-year-old female patient presented with rectorrhagia. A colonoscopy was found a sigmoid colic tumor later confirmed by CT (Figure 1) and surgery to be T3N0, with no liver ou lung metastasis. The patient had a 60 year/pack smoking history, but no specific complaints of chronic bronchitis. Chest CT made in the extension work-up showed complex alterations in the posterior basal segment of the right lower lobe (Figure 2(A)) just above the diaphragm while a ${ }^{18} \mathrm{~F}-\mathrm{FDG}$ $\mathrm{PET} / \mathrm{CT}$ scanner made for the colic tumor showed no uptake in the lung right lower lobe condensation (Figure 2(B) and Figure 2(C)).

Thin slices oblique reconstructions of Figure 3 demonstrate that there is some collapsed lung parenchyma around small tubular bronchiestasis that have a completely different orientation than the normal bronchial tree (Figure 3(B) and Figure 3(D)). They are roughly horizontal, parallel to the diaphragm, not connected to the normal bronchial tree and have a different orientation. Adjacent and medial to it, a $6.5 \mathrm{~mm}$ wide large systemic artery with an anterior-posterior direction, coming up from below the diaphragm through a defect between the main right diaphragmatic pillar (star) and the right accessory pillar. Contrary to all ILS described in the literature, the proximal part of that large systemic artery is not as large and regular than its distal intra-pulmonary part: it seems to be formed by the convergence of many very small serpiginous arteries coming up from the area of the celiac trunc and the common hepatic artery. So there is a "rete mirabile" (RM) composed of many small arteries converging up through a small hiatus in the diaphragm to reunite into one large systemic trunk of the right ILS (Figure 4(C), Figure 4(D), Figure 5(A) and Figure 5(D)).

There were no pulmonary symptoms related to the ILS (no dyspea, coughing, hemoptysis), so that no treatment was proposed for the ILS. The colic tumor was resected, the staging was PT3N0M0 on the basis of the histological findings, the absence of invasion of the 14 resected lymph nodes and the otherwise normal ${ }^{18}$ F-FDG PET. No chemotherapy was given as there were at this time no high risk factor of recurrence.

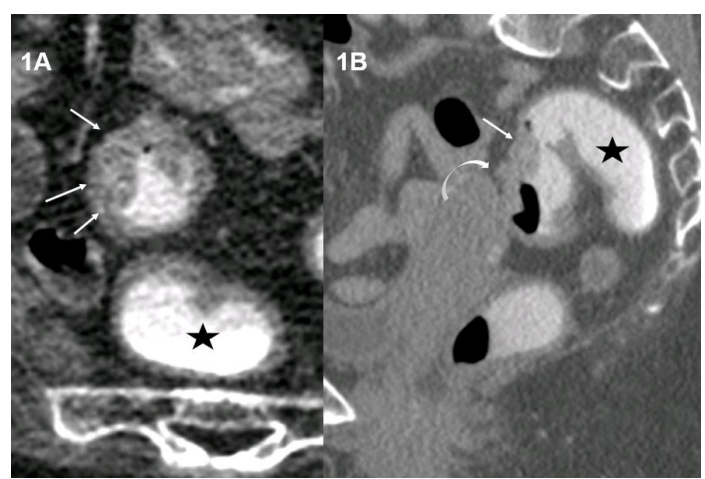

Figure 1. 1(A) Axial CT slice, with intra-rectal contrast (star). 1(B) Sagittal reconstruction. The distal sigmoid colon tumor is shown by the thin arrows and the curved arrow indicates tumor effraction through the serosa, it proved to be a T3N0M0 tumor. 


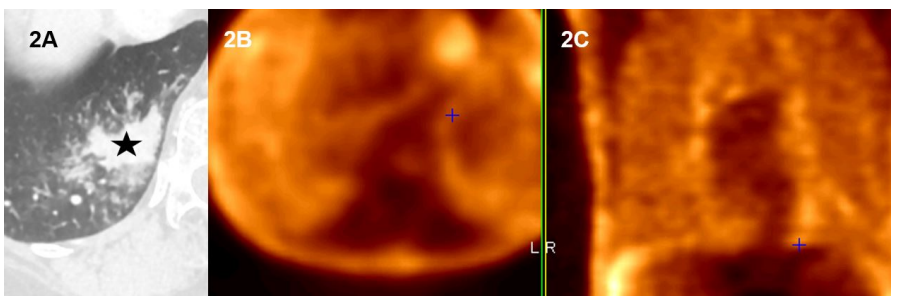

Figure 2. 2(A) axial CT through the right posterior-basal lung segment. 2(B) and 2(C) are ${ }^{18} \mathrm{~F}-\mathrm{FDG}$ PET/CT axial and coronal images through the same region. The lung postero-basal lesion (star) has irregular borders, On 2(B) and 2(C), there is no significative ${ }^{18} \mathrm{~F}-\mathrm{FDG}$ uptake.

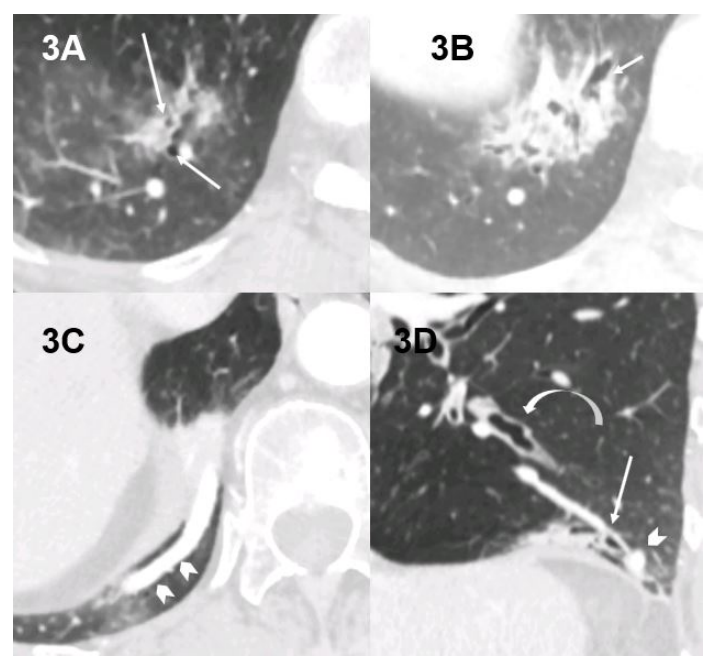

Figure 3. CT axial slices, at the level of the lung condensation (3(A), 3(B) and 3(C)), 3(D) sagittal reconstruction. The bronchi (thin arrows) inside the lesion above the diaphragm are irregular and dilated, they have a very different orientation than the proximal bronchi (curved arrow). On 3(C) and 3(D) is the very large systemic artery (arrowheads).

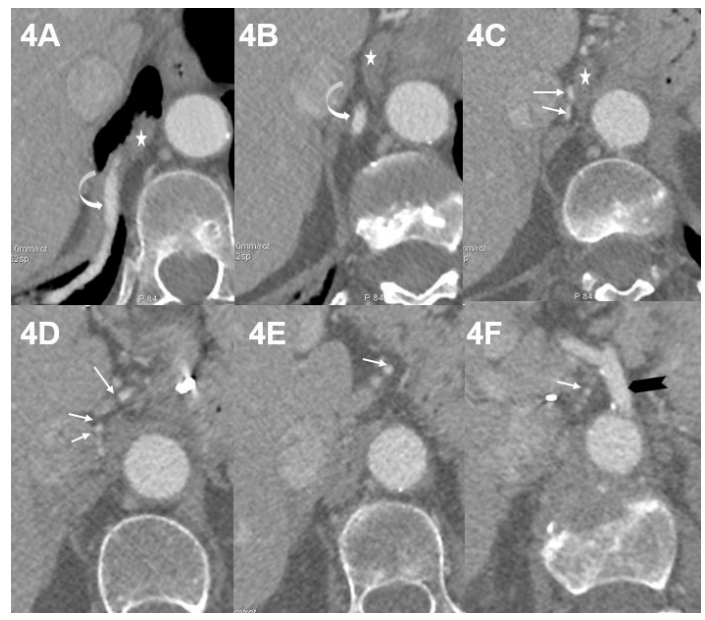

Figure 4. Abdominal CT Axial slices from the ILS 4(A) above to the celiac trunk below (black arrowhead in $4(\mathrm{~F})$. A series of very small arteries arising from the celiac area (thin arrows) converge to reunite into the large systemic artery (curved arrow) of the ILS. They pass through a gap in the diaphragm on the right side of its right pillar (star in 4(B) and 4(C)). 


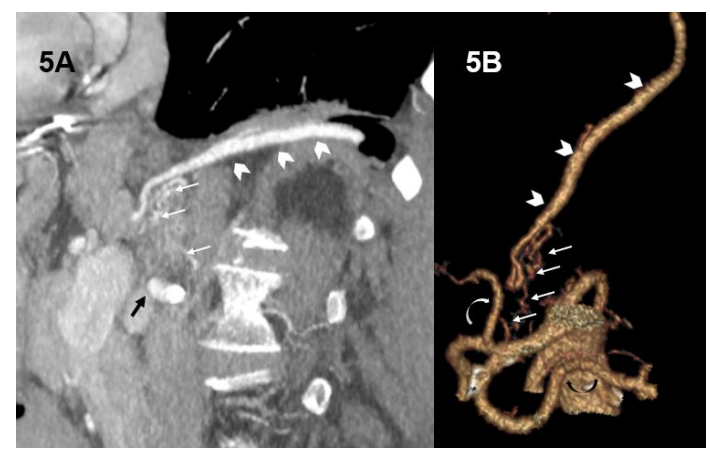

Figure 5. 5(A) is a MIP oblique sagittal reconstruction above the common hepatic artery (black arrow), showing the thin trabeculated arteries (thin arrows) going up from the region of the celiac trunc and hepatic artery to merge into the large systemic vessel (large arrowheads) which enters the ILS. 5(B) is a Volume Reconstruction from the angio CT. Hepatic artery (white curved arrow), splenic arteric (black curved arrow), rete mirabile (thin white arrows), large systemic vessel of the ILS (large arrowheads).

Two years later, a liver metastasis was detected in segment 2 and treated by left hepatectomy followed by FOLFOX chemotherapy for 6 months. Five years later two new liver metastases were found in segment 8 . The patient received 3 cycles of FOLFOX associated with PANitumumab followed by 1 cycle of PANitumumab alone which achieved partial response on the liver lesions that were resected with R0 margins. Since that last surgery, the patient receives adjuvant TOMOX chemotherapy (Raltitrexed plus oxaliplatin without $5 \mathrm{FU}$ ) at a $20 \%$ reduced dosage and one year later, MRI and CT exams do not show any no recurrence. During those 8 years, the ILS of the right pulmonary lobe did not change and the patient presented no specific symptoms that could be related to it.

\section{Discussion}

Pulmonary sequestration (PS) is a rare congenital malformation that accounts for $0.15 \%-6.4 \%$ of all pulmonary malformations [1]. In the year 1946, Pryce was the first to discribe the entity [2], which is an area of non-functioning bronchopulmonary tissue that is separated from the tracheobronchial tree and receives its arterial blood supply from the systemic circulation. The aberrant systemic arterial supply may involve a single or multiple vessels, and the latter accounts for $15 \%-20 \%$ of pulmonary sequestration [1].

By definition, there is no communication of the lesion with the tracheobronchial tree. Pulmonary sequestration can be divided into two distinct groups based on the relationship of the aberrant segmental lung tissue to the pleura: the intralobar sequestration (ILS) which accounts for the majority (75\% - 85\%) of all sequestrations and present later in childhood with recurrent infections [2]. ILS is closely connected to the adjacent normal lung and does not have a separate pleura while the less common extralobar sequestration (ELS) is separated from any surrounding lung by its own pleura. ELS usually is present in the neonatal period with respiratory distress, cyanosis, or infection, with a recognized male 
predilection, and infradiaphragmatic location in $10 \%$ of cases [3].

The most accepted theory concerning its pathogenesis suggests that PS is the result of an insult to the tip of developing lung buds. In early developing stages, the tips of the dividing bronchial buds are supplied by systemic capillary plexus derived from the primitive aorta. This plexus regresses with further lung maturation as the developing pulmonary artery takes over [4]. If an insult to the developing lung buds occurs, the original vessels derived from the aorta keep existing and that explains the presence of the systemic feeding vessel of the PS.

Imaging studies for the investigation of a suspected case of pulmonary sequestration have two principal objectives: to rule out other pathologies and to confirm the presence of an anomalous arterial supply. Both magnetic resonance imaging and CT are effective in identifying that aberrant arterial supply and, thus, establishing the diagnosis of ILS. But, given its ability to better simultaneously visualize the arterial supply, pulmonary parenchyma and venous drainage, multidetector CT angiography is probably the diagnostic test of choice [5] [6].

The abnormal systemic arterial supply was studied in a very large series [7]: the arterial supply of PS mainly originated from thoracic aorta (76.55\%) and abdominal aorta (18.47\%) with a few cases from the intercostal artery, diaphragmatic artery, left gastric artery and celiac trunk while the venous drainage of pulmonary sequestration was mainly to pulmonary veins (90.97\%).

The aberrant systemic arteries of ILS are usually large in diameter and histological analysis showed a preponderance of elastic vessels walls which helps to prove the congenital features of anomalous arterial supplies, as well as to distinguish it from bronchial arteries [5]. In no published case was mentioned a meshwork of tiny arteries converging into the large systemic feeding vessel of the ILS (Figure 5(A), Figure 5(B), Figure 6(B)), a rete mirabile (RM).

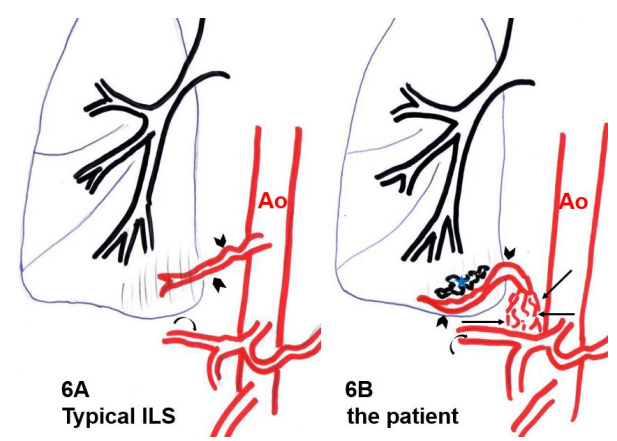

Figure 6. Drawing of two cases of a ILS (hatched area) in the right lower lobe. 6(A) is a typical case, with a large systemic artery coming from the aorta. 6(B) is a schematic drawing of our case. There is a large systemic vessel in the ILS, but it's connections with celiac trunk and common hepatic artery are through a "rete mirabile", a bunch of many small irregular arteries converging up in the big ILS artery. Bronchi are in black, arteries in red, pleura in blue. Common hepatic artery (curved arrow), ILS systemic artery (arrowheads), rete mirabile (thin arrows), bronchectasis inside the ILS (blue star). 
Rete mirabile (wonderful net in Latin) is an arterial meshwork [8], a small but dense network of blood vessels formed by the breaking up of a larger vessel into branches that usually reunite into one trunk. Vascular retia mirabilia are encountered in birds, fishs and are also found in the limbs of a range of mammals. These reduce the temperature in the extremities. Some of these probably function to prevent heat loss in cold conditions by reducing the temperature gradient between the limb and the environment. In giraffes a rete mirabile in the neck equalizes blood pressure when the animal bends down to drink.

In normal human patients, RM was not described. The ancient physician Galien mistakenly thought that humans also have a rete mirabile in the neck, apparently based on dissection of sheep and misidentifying the results with the human carotid sinus. But Vesalius later demonstrated the error. A few cases of rete mirabile were described in human [8] [9] at the cavernous portion of the internal carotid artery (ICA) in patients with agenesis of the ICA. In those cases, it was considered to be a collateral pathway that develops as a consequence of segmental agenesis of the ICA [8]. An ophtalmic rete mirabile was also described in a patient with Moyamoya disease, a progressive, occlusive disease of the cerebral vasculature with early onset, the highest peak being in the first decade. This ophtalmic RM was also considered as embryonic collaterals [10].

On basis of the fact that all RM in human seem to be collateral pathways as a consequence of secondary arterial stenosis or occlusion, and that no other case of RM in ILS was described, we could evoke the hypothesis that a large vessel from the celiac trunk or hepatic artery to the ILS existed, that it thrombosed early and that the patient's RM could be a collateral pathway to revascularize the ILS. That hypothosis could also explain the paucity of symptoms of the ILS (due to the reduced arterial flow ...).

\section{Conclusion}

ILS is a congenital pathology where part of the lung, usually in the lower lobes, has no connection with the central airways and an arterial supply coming form a systemic origin and where the abnormal lung segment lies in the same pleural sac. The key point to diagnosis is to confirm the presence of an anomalous arterial supply and its origin, often the thoracic aorta, sometimes the abdominal aorta or one of its upper branches. This is best done by CT. An asymptomatic ILS was found in a cancer patient, with a large systemic artery coming up from below the diaphragm. This systemic artery was formed by the convergence of many very small serpiginous arteries coming up from the area of the celiac trunk and common hepatic artery: a kind of "rete mirabile". A hypothesis for this peculiar morphology is proposed: it could be formed by collaterals secondary to an early segmental thrombosis of that vessel.

\section{Conflicts of Interest}

The authors declare no conflicts of interest regarding the publication of this paper. 


\section{Informed Consent}

Informed consent was obtained from the patient.

\section{References}

[1] Hou, X.M., Li, J., Li, J. and Cai, B.Q. (2017) Anomalous Systemic Arterial Supply of Pulmonary Sequestration in Adult Patients. Annals of Thoracic Medicine, 12, 46-50. https://doi.org/10.4103/1817-1737.197778

[2] Dhingsa, R., Coakley, F.V., Albanese, C.T., Filly, R.A. and Goldstein, R. (2003) Prenatal Sonography and MR Imaging of Pulmonary Sequestration. American Journal of Roentgenology, 180, 433-437. https://doi.org/10.2214/ajr.180.2.1800433

[3] Wani, S.A., Mufti, G.N., Bhat, N.A. and Baba, A.A. (2015) Pulmonary Sequestration: Early Diagnosis and Management. Case Reports in Pediatrics, 2015, Article ID: 454860. https://doi.org/10.1155/2015/454860

[4] Pryce, D.M. (1946) Lower Accessory Pulmonary Artery with Intralobar Sequestration of Lung: A Report of Seven Cases. The Journal of Pathology and Bacteriology, 58, 457-467. https://doi.org/10.1002/path.1700580316

[5] Boyden, E.A. (1958) Bronchogenic Cyst and the Theory of Intralobar Sequestration: New Embryologic Date. Journal of Thoracic Surgery, 35, 604-616.

[6] Litt, D., Gandhi, S., Bhinder, S., Blitz, M. and McIntyre, K. (2013) Incidental Finding and Management of Intralobar Sequestration of the Lung in a 24-Year-Old Man. Canadian Respiratory Journal, 20, 403-405. https://doi.org/10.1155/2013/369161

[7] Wei, Y. and Li, F. (2011) Pulmonary Sequestration: A Retrospective Analysis of 2625 Cases in China. European Journal of Cardio-Thoracic Surgery, 40, e39-e42. https://doi.org/10.1016/j.ejcts.2011.01.080

[8] Verma, A., Pendharkar, H. and Prasad, C. (2018) Rete Mirabile of the ICA: Report of Three Cases. Interventional Neuroradiology: Journal of Peritherapeutic Neuroradiology, Surgical Procedures and Related Neurosciences, 24, 586-590. https://doi.org/10.1177/1591019918772054

[9] Şahin, H., Çınar, C. and Oran, I. (2010) Carotid and Vertebrobasilar Rete Mirabile: A Case Report. Surgical and Radiologic Anatomy, 32, 95-98. https://doi.org/10.1007/s00276-009-0531-x

[10] Chung, J.I. and Weon, Y.C. (2008) Ophthalmic Rete Mirabile: The First Angiographic Documentation of Embryonic Ophthalmic Collaterals in a Patient with Moyamoya Disease. Interventional Neuroradiology, 14, 293-296.

https://doi.org/10.1177/159101990801400309 